\title{
The observed neutral manganese line profiles in solar spots and plages
}

\author{
Olexa V. Andriyenko \\ IC AMER of NAS of Ukraine, 27 Zabolotnogo Street, Kiev, 03680, Ukraine \\ email: olexa@mao.kiev.ua
}

\begin{abstract}
From observations of the Sun as a star made at Kitt Peak it is known that MnI 539.5 $\mathrm{nm}$ line exhibits unusually big (for a photospheric line) variations during a solar activity cycle. Doyle et al. 2001 suggested that it is because of optical pumping. This work aims particularly to check this hypothesis with help of observations.
\end{abstract}

According to W. Livingston et al. (1987) Mn I $539.5 \mathrm{~nm}$ CI and EW change for more than $1 \%$ with a course of solar activity cycle. It is unusual behaviour for a photospheric line. One can find a nice review of the problem in I. Vince (2002). According to this review and references therein it is impossible to explain observable variations of $\mathrm{MnI}$ $539.5 \mathrm{~nm}$ neither with global variations of solar temperature nor with changing of its profile in plages and spots. Recently Doyle et al. (2001), using NLTE calculations, have shown that $\mathrm{MnI} 539.5 \mathrm{~nm}$ line is sensitive to the optical pumping of other manganese line. Namely the MnI uv1 line $(279.48 \mathrm{~nm})$ overlaps with the MgII k line $(279.5 \mathrm{~nm})$. As $539.5 \mathrm{~nm}$ and uv1 lines have the same lower excitation level, optical pumping from $\mathrm{MgII}$ $\mathrm{k}$ line decreases population of the level and $\mathrm{MnI} 539.5 \mathrm{~nm}$ line becomes weaker.

Observations were obtained at Terskol observatory (3100 m, Central Caucasus) with ATsU-26 telescope (see the instrument description on http://www.terskol.com). Lines with the same $(0.0 \mathrm{eV}$ for 539.5 and $543.3 \mathrm{~nm}$ lines) and with different $(2.14 \mathrm{eV}$ for $542.0 \mathrm{~nm}$ line and 3.07 for $601.7 \mathrm{~nm}$ line) lower excitation levels were chosen. Each line in any position at the solar disk was observed for at least $15 \mathrm{~min}$ with $15 \mathrm{sec}$ cadence with aim to exclude influence of 5 min oscillations. Each CCD image in an observational sequence was then corrected for dark current, flat field and scattered light level. Profiles were normalized to continuum level and then corrected for instrumental profile. Results are presented on figures 1 and 2 .

On the figures it is possible to see that all the lines are stronger in spots and weaker in plages. Similar results for some of the lines were obtained by O. Malanushenko et al. (2004), I. Vince (2002). From figures 1 and 2 one can also note an influence of hyperfine structure on the line profiles. The optical pumping hypothesis predicted that 539.5 and $543.2 \mathrm{~nm}$ lines should be weaker in plages. Indeed this is confirmed with observations. But other manganese lines also demonstrate such an effect. Without accurate modelling it is difficult to confirm or reject the hypothesis with these observational results.

It is worth to point out that observations of the spot in AR0087 are standing apart of other spot observations. In that spot both 539.5 and $543.2 \mathrm{~nm}$ lines were stronger than in other spots, but $539.5 \mathrm{~nm}$ line was almost saturated. Furthermore $539.5 \mathrm{~nm}$ line was unusually strong at the spot edge and in the vicinity of the active region (about 100 arcsec apart), while $543.2 \mathrm{~nm}$ line was not stronger in those places than in such places near other spots. I cannot suggest any cause for this observational fact. This is also difficult to adjust with the optical pumping hypothesis. 

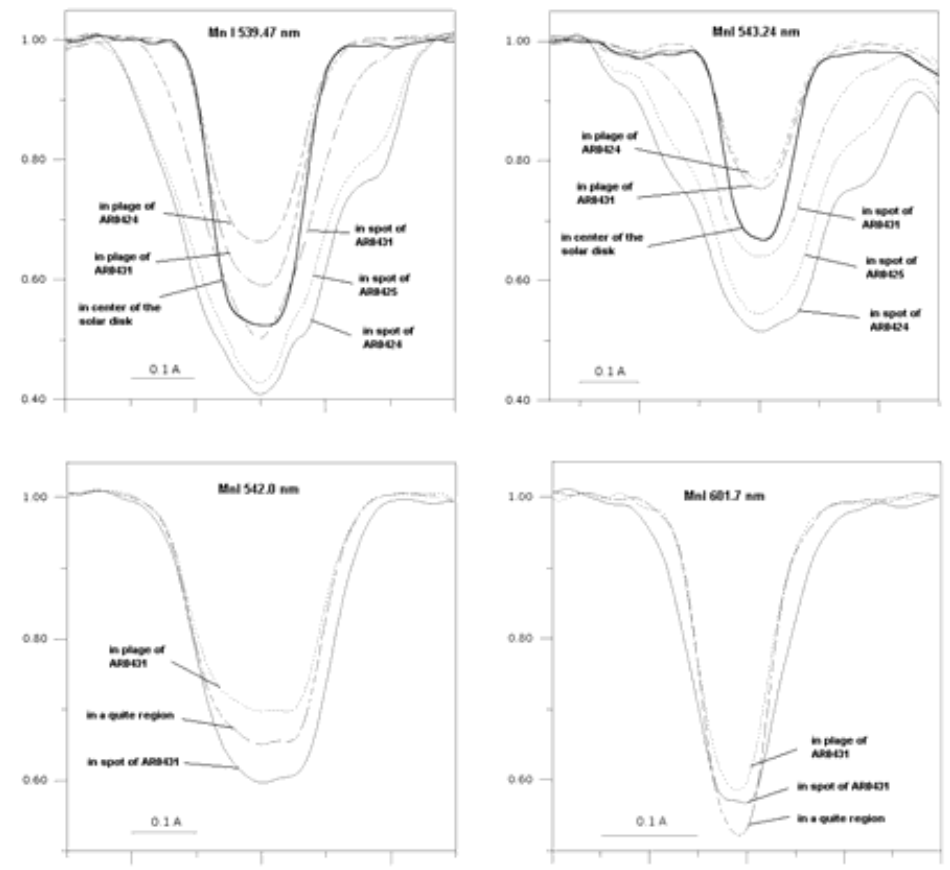

Figure 1. Profiles in spots and plages of AR0424, AR0425 and AR0431. Observations were made on 7, 13 and 14 August of 2003
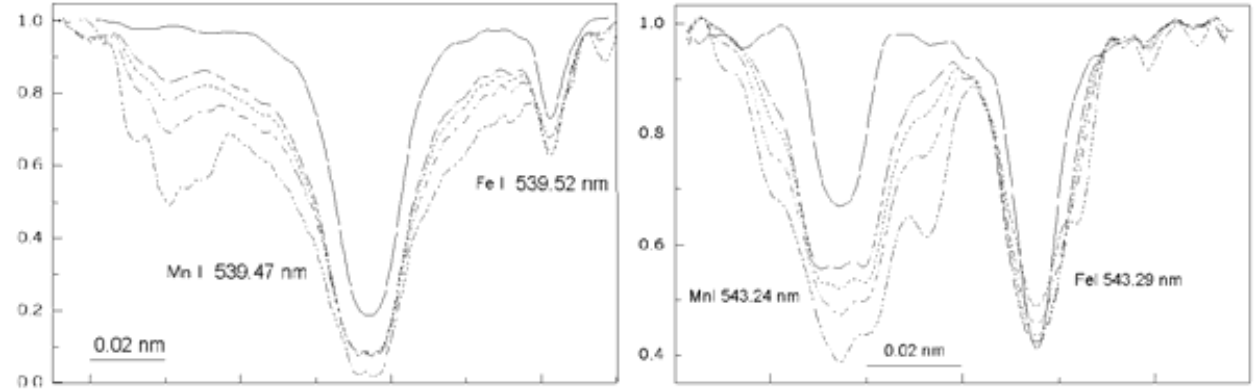

Figure 2. Profiles in penumbra and umbra of the preceding spot of AR0087. The solid lines profiles in vicinity of the spot. Observations were made on 25 August of 2002

\section{References}

Doyle, J. G., Jevremovic, D., Short, C. I., Hauschildt, P. H., Livingston, W. and Vince, I. 2001 Astron. Astrophys 369, L13.

Livingston, W. and Wallace, L. 1987 Astrophys. J. 314, 808.

Vince, I. 2002 in "Solar Researches in the South-Eastern European Countries: Present and Perspectives" (eds. G. Maris and M. Messerotti), Editions de l'Academie Roumaine, 139.

Malanushenko, O., Livingston, W. and Jones, H. 2004 IAU Symposium 223 (book of abstracts), 140. 